\title{
Irish Nursing Students Perceptions of a Technology Enhanced Blended Approach to Teaching and Learning
}

\author{
Kevin Johnson, Claire O Donnell, and Kathleen Markey \\ Dept of Nursing and Midwifery, University of Limerick, \\ Limerick, Ireland
}

Kevin.Johnson@ul.ie Claire.ODonnell@ul.ie Kathleen.Markey@ul.ie

\author{
Brian Lake \\ Unit for the Enhancement of Learning and Teaching, University \\ of Kent, Canterbury, UK \\ b.lake@kent.ac.uk
}

\begin{abstract}
Health informatics is becoming increasingly important for healthcare professionals and strategies to promote health informatics development are becoming increasingly popular in undergraduate professional programmes. With this in mind, a blended or hybrid learning approach was piloted with an undergraduate BSc nursing module in the university. The aim of this pilot was to design, develop, implement and evaluate the students' perceived reactions to learning, teaching and assessment incorporating several online components. Blended learning is the combination of multiple approaches to learning such as a combination of technology-based materials and face-to-face sessions used together to deliver instruction. A branded version of Sakai (the university's Learning Management System [LMS]) was used. The students interacted with the system on a weekly basis and their perceptions were codified with the aid of an online questionnaire based on the preferred COLLES format. Additionally, a touch screen based recording suite was availed of permitting the students to record themselves performing a task and later review the footage. The initial findings appear to provide a positive outlook towards the use of technology within the course and the potential for further expansion in the near future.
\end{abstract}

Ke ywords: Blended, Learning, Technology Enhanced Learning, Perception, LMS, Video Recording, Teaching 